\title{
Multidimensional approach for the proper management of a complex chronic patient with chronic obstructive pulmonary disease
}

\author{
Paola Rogliani, Vito Brusasco, Leonardo Fabbri, Andrea Ungar, Elisa \\ Muscianisi, Ilaria Barisone, Alberto Corsini \& Giuseppe De Angelis
}

To cite this article: Paola Rogliani, Vito Brusasco, Leonardo Fabbri, Andrea Ungar, Elisa Muscianisi, llaria Barisone, Alberto Corsini \& Giuseppe De Angelis (2017): Multidimensional approach for the proper management of a complex chronic patient with chronic obstructive pulmonary disease, Expert Review of Respiratory Medicine, DOI: 10.1080/17476348.2018.1417041

To link to this article: https://doi.org/10.1080/17476348.2018.1417041

Accepted author version posted online: 15

Dec 2017.

Submit your article to this journal $₫$

View related articles ¿

View Crossmark data $₫$ 
Publisher: Taylor \& Francis

Journal: Expert Review of Respiratory Medicine

DOI: $10.1080 / 17476348.2018 .1417041$

Review

Multidimensional approach for the proper management of a complex chronic patient with chronic obstructive pulmonary disease

Paola Rogliani ${ }^{*}$, Vito Brusasco ${ }^{2}$, Leonardo Fabbri ${ }^{3}$, Andrea Ungar ${ }^{4}$, Elisa Muscianisi ${ }^{5}$, Ilaria Barisone $^{5}$, Alberto Corsini ${ }^{6,7}$, and Giuseppe De Angelis ${ }^{8}$

${ }^{1}$ Experimental Medicine and Surgery Dept, University of Rome "Tor Vergata”, Italy ${ }^{2}$ Internal Medicine Dept, University of Genoa, Italy

${ }^{3}$ Endocrinology, Metabolism and Geriatric Dept, Sant'Agostino Hospital, Modena, Italy

${ }^{4}$ Geriatric and Intensive Care Medicine, AO Careggi and University of Florence, Italy,

${ }^{5}$ Novartis Farma Spa, Respiratory Franchise, Origgio (VA), Italy,

${ }^{6}$ Pharmacological and Biomolecular Sciences Dept, University of Milan, Italy

${ }^{7}$ Multimedica IRCCS, Milan Italy

${ }^{8}$ Cardiovascular Dept, University of Milan, Italy.

* Corresponding author:

Professor Paola Rogliani

Department of Systems Medicine

University of Rome "Tor Vergata"

Via Montpellier, 1 -00133- Rome, Italy

Email: paola.rogliani@uniroma2.it 


\begin{abstract}
Introduction: Chronic obstructive pulmonary disease (COPD) is frequently associated with comorbidities occurring either independently or as consequences of COPD.
\end{abstract}

Areas covered: This review examines the interactions between the pathophysiology of COPD and the most frequent comorbidities, and highlights the need for multidimensional clinical strategies to manage COPD patients with comorbidities.

Expert commentary: Most COPD patients need to be approached in a complex and multifactorial scenario. The diagnosis of COPD is necessarily based on the presence of chronic respiratory symptoms and poorly reversible airflow obstruction, but exacerbations and comorbidities need to be considered in the evaluation of disease severity and prognosis in individual patients. More importantly, defining the precise relationship between COPD and comorbidities for each patient is the basis for a correct therapeutic approach.

Keywords: COPD, ageing, cardiovascular diseases, comorbidity, risk factors, smoking. 


\subsection{Introduction}

Ever since the pioneering study by Burrows et al. [1], chronic obstructive pulmonary disease (COPD) has been considered as a condition characterized by airflow obstruction due to airway and parenchymal abnormalities [2]. During the first decade of the current century, despite the fact that the Global Initiative for Chronic Obstructive Lung Disease (GOLD) committee had given more emphasis to putative inflammatory mechanisms, the role of the different structural changes in the airways and pulmonary parenchyma in the pathogenesis of airflow obstruction was overshadowed [3]. Thus, several changes have been proposed in the definition of COPD, the most striking of which was in 2007, when airflow obstruction was considered as the pulmonary component of a systemic inflammatory multi-morbid disorder [4]. Notwithstanding, the demonstration of airflow obstruction by spirometry was reasonably retained to confirm a clinical diagnosis of COPD. Finally, the GOLD committee in 2017 went back to the definition of COPD as a "disease that is characterized by persistent respiratory symptoms and airflow limitation due to airway and/or alveolar abnormalities", where comorbidities are considered as frequently associated conditions [5].

COPD is characterized by a slowly progressive deterioration in lung function, mostly becoming evident with aging. Therefore, it is not surprising that COPD is frequently associated with other age-related morbid conditions, which may exist independently, or as consequences of COPD, or because they share common risk factors.

The aims of this review are to examine the interactions between the pathophysiology of COPD and the most frequent comorbidities, and to highlight the need for multidimensional clinical strategies to manage COPD patients with comorbidities by suitable diagnostic procedures and individualized therapeutic interventions. 


\subsection{Pathophysiology of COPD and related comorbidities}

Tobacco smoking and environmental exposures are the main risk factors for COPD. The exposure to tobacco smoking, ever since antenatal period and early childhood, and the presence of other childhood risk factors, such as maternal and paternal asthma, childhood asthma, and respiratory infections, accelerate the rate of lung function decline and COPD [6]. Cohort studies indicated that subjects with COPD in the sixth decade already had the worst spirometry at age 10 years, when spirometry was performed from the preschool years to late middle age [6]. Therefore, COPD seems to have its roots in early life; then, the individual susceptibility associated with genetic predisposition, abnormal lung development, and accelerated ageing may contribute to COPD development. Putative genetic risk factors for COPD include the alpha-1 antitrypsin deficiency, alterations in matrix metalloproteinases 12 (MMP12), alpha nicotinic acetylcholine receptor, and hedgehog interacting protein genes $[7,8]$. Ageing is also considered as a risk factor but its contribution in the COPD pathogenesis is not completely understood [5].

The pathology of COPD includes alterations of airways, lung parenchyma, and pulmonary vasculature. An increased numbers of specific inflammatory cell types (CD8+ Tc1 lymphocytes, neutrophils, and macrophages) in the lung due to noxious exposures may contribute to chronic inflammation, while structural changes (remodeling) result from repeated injury and repair [9]. These changes, which are considered the perpetrators of loss of lung function, do not always occur together, but evolve at different rates over time [5]. Oxidative stress, protease-anti-protease imbalance, chemotactic factors, cytokines, and growth factors promote and sustain inflammation, which leads to direct damage of airways (chronic bronchitis) and lung parenchyma (emphysema), and hence to systemic consequences [10-12]. Systemic inflammation contributes to skeletal muscle wasting and cachexia, and initiates or worsens comorbidities [5]. 
Recently it has been shown that COPD-associated airflow obstruction or emphysema reduce the left ventricular function and affect cardiac stroke volume [13]. Airflow limitation contributes to the positive intrathoracic pressure during expiration, while the loss of alveolar septa reduces pulmonary vascular volume [13]. Therefore, altered heart-to-lung mechanical interactions may affect the cardiac function, for instance determining right heart dysfunction secondary to pulmonary hypertension in hypoxemic patients.

\subsection{The pulmonary profile}

Although it has been recognized that COPD is a heterogeneous condition with respect to the underlying pulmonary pathology, current documents [5] and most guidelines [14-16] recommend the use of simple spirometry to diagnose COPD and grade its severity. This simplistic approach may lead to misclassification of several COPD subjects for a series of reasons.

In early phases, abnormalities are limited to peripheral airways and need to be considerable to affect overall lung function tests, such as spirometry [17]. Moreover, the forced expiratory flow is an integrated measure of maximal expiratory flows of individual airways, which are determined by their caliber, stiffness, and elastic recoil pressure. Simple spirometry is not sensitive to evaluate regional inhomogeneity and does not allow distinguishing between airway and parenchymal components of airflow obstruction. For instance, increased total lung capacity and reduced single-breath diffusion capacity for carbon monoxide are sensitive to recognizing emphysema [18], but these tests are not considered as necessary in the routine COPD management [5].

Furthermore, the fixed ratio FEV1/FVC $<0.7$ is a commonly used criterion in clinical practice to define airflow obstruction as per the first GOLD recommendation [3] and to diagnose COPD [19-21]. However, it presents some age and sex biases, which may lead to 
over-diagnosis in older subjects and males and under-diagnosis in younger subjects and females.

In the evaluation of predicted FEV1, some confounding factors related to lung pathology or comorbidities should be kept into account: despite similar symptoms, FEV1 may be significantly lower in patients with dominant emphysema than in those with chronic bronchiolitis, due to thoracic gas compression [22]. In obesity, the ratio FEV1/FVC tends to increase with the body mass index (BMI), whereas FVC and FEV1 are reduced [23]. Thus, in obese COPD subjects the presence of airflow obstruction may go undetected, but its severity may be overestimated, if based on FEV1 [24,25].

The reduction of FEV1 is not the unique relevant abnormality of lung function in COPD. Considering the simple relationship FEV1 $=$ FVC $x$ FEV1/FVC, FEV1 reduction is not only due to airway narrowing or low elastic recoil pressure (low FEV1/FVC), but also to air trapping (low FVC). Since more than 50 years, it was known that air trapping is present in smokers before FEV1 or FEV1/FVC decrease [26,27]. Therefore, based on simple spirometry, some COPD subjects may be paradoxically classified as restricted [28]. In these cases, an additional investigation of absolute lung volumes may be useful for a correct functional classification [29].

Furthermore, spirometry is a diagnostic test based on a forced expiratory maneuver, that is not commonly experienced in the daily life: the spirometric measurements are good survival predictors [30], but loosely correlated with patient-centered outcomes, such as dyspnea, quality of life, exercise tolerance [31], that are more closely related to lung hyperinflation than FEV1 [32].

Imaging techniques may provide quantitative information on the extent of emphysema and airway wall abnormalities [33]. However, recent data have indicated that 
combining clinical and functional data allows distinguishing patients with prevalent emphysema or prevalent chronic bronchitis, with an accuracy like that of high-resolution computerized tomography [34].

\subsection{The Co-morbidome}

Comorbidities frequently occur in patients with COPD, and the frequency of comorbidities is independent of the degree of airflow limitation [35]. Comorbidities contribute to adverse clinical outcomes in these patients and affect symptom burden, functional performance, hospitalization risk, and mortality. In randomized controlled trials, comorbidities are the primary causes of death in more than $60 \%$ of non-survivors $[36,37]$.

Several algorithms have been proposed to predict the relationship between comorbidities and COPD clinical outcomes. The COPD specific CO-morbidity Test (COTE) is a point scale index, based on prevalence (more than 10\%) of individual comorbidities (congestive heart failure, coronary artery disease, atrial fibrillation, pulmonary fibrosis, lung cancer, anxiety, breast cancer, pancreatic cancer, esophageal cancer, diabetes/ neuropathy, gastric duodenal ulcer, liver cirrhosis) and their association with mortality risk overtime [38]. Vanfleteren et al. [39] profiled five comorbidity-based clusters: less morbidity cluster (cluster 1), cardiovascular cluster (cluster 2), cachectic cluster (cluster 3), metabolic cluster (cluster 4), psychological cluster (cluster 5) [39]. Assigning each patient to a specific cluster or determining the COTE index may be important for the overall management of patients.

In the overall management of COPD patients, the assessment of comorbidities is a valuable tool to improve prognosis and quality of life because COPD and comorbidities synergize to define the health status. 


\subsection{Existing evidence for therapeutic management}

The therapeutic management of COPD should consider the patient as a whole and all interventions should be aimed to improve the overall health status. The worsening of COPD in patients with comorbidities may affect clinical outcomes and prognosis; therapies should be evaluated not only based on their safety and efficacy, but also on their ability to prevent exacerbations. However, it should be kept into account that COPD treatment may improve symptoms and decrease exacerbations, but does not prevent progression nor increase survival [40]. By contrast treatment of comorbidities may prolong survival and decrease health care utilization. Therefore, it is extremely important to promptly diagnose and treat comorbidities in these patients.

Among non-pharmacological interventions, smoking cessation is a must to reduce the rate of lung function decline; vaccination against influenza is demonstrably linked to reduced exacerbations and mortality; pulmonary rehabilitation and interventions to increase physical activity are even recommended to improve the overall health status [5,33].

Therapeutic options for COPD include $\beta 2$-agonist and anticholinergics as bronchodilators, eventually associated with inhaled corticosteroids and phosphodiesterase-4 inhibitors or macrolides for exacerbations. Among bronchodilators, $\beta 2$-agonists (salmeterol, formoterol, indacaterol, salbutamol, olodaterol, vilanterol) and anticholinergics (ipratropium bromide, oxitropium bromide, aclidinium bromide, tiotropium bromide, glycopyrronium bromide, umeclidinium bromide) are associated with functional improvement of lung function, symptom control, and quality of life [41-45].

Clinical guidelines and strategical plans for COPD management indicate that corticosteroids combined with long-acting $\beta 2$-agonist are useful to reduce the effects of chronic inflammation, since they may contribute to improve lung function, delay disease 
progression, and decrease symptoms and exacerbations [5,33]. However, the results of FLAME study indicate that the combination of long-acting $\beta 2$-agonist (LABA) and longacting muscarinic receptor antagonist (LAMA) was superior to the combination of LABA and inhaled glucocorticoid in ameliorating exacerbations, lung function, and health status [46]. The effect was independent on blood eosinophil levels that did not correlate with the rate of moderate or severe exacerbations [47].

Phosphodiesterase-4 inhibitors are other anti-inflammatory agents, which inhibit the breakdown of intracellular cyclic AMP; they do not have direct bronchodilator activity, but improve FEV1 in patients treated with LABA and LAMA and reduce moderate and severe exacerbations with concomitant corticosteroids [5,48,49]. Long term treatment with macrolides may reduce exacerbations in severe COPD patients not controlled by inhalation therapy [50].

Considering that COPD is a complex disease based on low grade systemic inflammation, it is also possible to target abnormal inflammation, either locally at pulmonary level or systemically. Some evidences indicate that pulmonary inflammation due to tobacco smoking not only leads to the structural changes observed in COPD, but also contributes to extrapulmonary systemic inflammation and comorbidities: smoking cessation and bronchodilators decrease dynamic hyperinflation and, therefore, reduce systemic inflammation [51]. Plasmatic CRP is increased in COPD patients and seems to contribute to atherosclerosis: based on this observation, inhaled corticosteroids (ICS) may potentially reduce the risk of cardiovascular diseases in COPD patients [12]. However, results on survival and risk of myocardial infarction are controversial and no evidence supports a negative relationship between ICS and mortality or cardiac adverse events in COPD patients, with or without concomitant cardiovascular disease [52]. 
COPD patients present with a variable mix of distinct phenotypes such as emphysema, bronchitis, small airways disease, frequent vs infrequent exacerbators, or those with a rapid decline in lung function, which are independent of genetic background [53-54]. Including inflammatory markers in the definition of COPD sub-phenotype would enable to identify those patients who could benefit from more personalized approach in the future [41]. Targeting oxidative stress is another possible way to treat COPD. In COPD patients, glutathione (GSH) is increased to counter excess oxidant production; $\mathrm{N}$-acetyl-1-cysteine acts as a precursor during GSH synthesis and it is currently used as a mucolytic and antiinflammatory agent in chronic bronchitis [56-58]. In recently published evidence-based guidelines on the prevention of acute exacerbations of COPD by American College of Chest Physicians and Canadian Thoracic Society, N-acetylcysteine therapy is recommended (grade 2B) for patients with moderate to severe COPD and a history of two or more exacerbations in prior 2 years [59].

\subsection{COPD and cardiovascular diseases}

The diagnostic challenge is to determine how COPD and comorbidities specifically contribute to the disease, when respiratory symptoms (dyspnea, cough, or wheezing) are overlapping, as in patients with decompensated heart failure, arrhythmias, and ischemic heart disease. On one hand, airway and lung inflammation contributes to a systemic inflammation which involves vessels, heart, and central nervous system and worsens concomitant cardiovascular or metabolic diseases; on the other, comorbidities (i.e. acute myocardial infarction, congestive heart failure, cerebrovascular disease, cardiac arrhythmias and pulmonary circulation disorders) produce acute events and affect morbidity and mortality during COPD exacerbations [60].

Worldwide in general population, COPD prevalence ranges from 7 to $10 \%$, and is 
continuously increasing. In the US and Europe, COPD is the fourth cause of death. Ischemic heart disease with all its clinical manifestations, including silent ischemia, sudden death, angina, myocardial infarction, is the first cause of death in developed countries, and it will become soon the first in developing ones $[61,62]$. Therefore, it is presumable that COPD and ischemic heart disease frequently coexist in patients. In almost one third of patients, COPD is associated with cardiovascular diseases [63], which are ischemic heart diseases in a half of the patients [64]. The prevalence of ischemic heart disease in patients with COPD is double compared with that in general population [65]. COPD may induce ischemic heart disease or, at least, increase the risk for developing it, and it has been demonstrated that cardiovascular diseases and mortality correlate with pulmonary failure severity [66]. COPD and atherosclerosis, which foreshadows ischemic heart disease, share a similar pathogenesis, the smoking habit as major risk factor, and an increased level of many markers of systemic inflammation, including $\mathrm{C}$ reactive protein, IL-8, TNF- $\alpha$, and fibrinogen. Three among these variables -age $>40$ years, smoking habit, COPD, heart failure, metabolic syndrome, high PCR levels- allow identifying patients with systemic chronic inflammation [67]. A case-control study with 11155 patients indicated that there was a strong relationship between pulmonary infection and incidence of first myocardial infarction within 1-4 weeks after the acute event [68]. Furthermore, anti-inflammatory agents, as phosphodiesterase 4 inhibitors, in addition to usual COPD therapy improved pulmonary function [69] and reduced exacerbations [70].

Identifying concomitant COPD and heart failure may be challenging, with serious diagnostic and therapeutic consequences: a missed or delayed diagnosis of both conditions leads to under-evaluation and under-treatment of symptoms, wrongly attributed only to a disease, and complicates the therapeutic approach [71]. Patients with COPD had high prevalence of heart failure, as they are at highest risk for cardiovascular morbidity and mortality, regardless other risk factors. Almost 10-40\% patients with heart failure are affected 
by COPD, and among patients with COPD, the prevalence of heart failure and chronic pulmonary heart is almost $20 \%$ and $2 \%$, respectively. Therefore, it is not surprising that the main cause of mortality in COPD patients is heart failure, and not respiratory failure [72], and it may be precautionary to manage patients with concomitant COPD and heart failure, as in presence of ventricular dysfunction until a different evaluation.

Sometimes, COPD is the primary cause of cardiovascular diseases, i.e. in case of pulmonary heart. All morbidities which either directly or indirectly affect respiratory mechanical ventilation, gas exchanges, and pulmonary vascular bed, may determine pulmonary hypertension and chronic pulmonary heart. Pulmonary hypertension accounts for $5-10 \%$ of all organic heart diseases and is reported in $20-30 \%$ of patients hospitalized for heart failure, who frequently are males, smokers, and 50-60 years old. There are many pathogenic mechanisms underlying pulmonary hypertension that, however, becomes evident when the vascular reserve is extremely limited and chronic. The primary pulmonary disease usually has a limited extension or do not affect gas exchanges and, therefore, do not trigger the process that leads to right ventricular hypertrophy and heart failure.

Chronic cardiovascular comorbidity of COPD should be accurately investigated and evaluated for an appropriate diagnosis. Electrocardiogram, echocardiogram and biomarkers, such as N-terminal pro-brain natriuretic peptide (NT-probing) and troponin, may help to specify the diagnosis, but abnormalities of biomarkers or imaging are frequently observed also in COPD patients without over clinical manifestations of myocardial damage and/ or dysfunction [60]. The search of reliable biomarkers of respiratory symptom exacerbation is an unmet need that should be rapidly solved. Furthermore, an early identification of cardiovascular comorbidities in COPD patients and a prompt therapeutic treatment of singular disease may improve quality of life and prognosis of these patients [40]. These 
observations are currently extrapolated from registries, observational studies or post-hoc analyses from clinical trials, but randomized clinical trials specifically designed to investigate the contribution of comorbidities in patients with COPD are lacking [40].

\subsection{Management of cardiovascular disease concomitant with COPD}

Both acute and chronic lung inflammation contribute to cardiovascular morbidity in COPD patients $[73,74]$. Chronic lung and systemic inflammation results in increased plaque cell turnover and oxidative stress, thus leading to an accelerated plaque progression, development and rupture in the ischemic heart disease or carotid arterial disease (CAD) [73]; acute lung inflammation is mainly associated with acute coronary syndrome [74]. Clinicians managing patients with a significant smoking history or occupational or home exposure to dust and particles should evaluate both cardiovascular and respiratory systems for abnormalities. An algorithm to predict the risk of CAD in COPD patients has been proposed, using a non-invasive approach. The model was developed on 1596 patients undergoing coronary arteriography: gender (men/women OR: 1.7, 95\%CI: 1.237-2.5, P < 0.05), dyslipidemia (OR: 1.8, 95\%CI: 1.2-2.5, P < 0.01), and smoking habit (OR: 1.5, 95\%CI: $1.2-$ $1.9, \mathrm{P}<0.001)$ were significantly associated with CAD in COPD and could specifically identify patients at risk with a high diagnostic accuracy [75].

Cardiovascular safety should be monitored during COPD therapy for many reasons: both LAMAs and LABAs have a high potential to impact on cardiovascular activity [52]; COPD per se increases the cardiovascular risk; some patients may be on long-term treatment for previous cardiovascular diseases.

Cardio- and cerebrovascular (CCV) safety is investigated during clinical developmental program, as a part of the comprehensive evaluation of safety [46]. A comparative analysis of safety data from phase III pivotal studies of at least 6 months 
duration indicated that indacaterol, formoterol and salmeterol were not associated with a significant increase in relative risk of serious CCV events, compared to placebo. For its intended long-term regular use, CCV safety of indacaterol at highest doses $(600 \mu \mathrm{g})$ was evaluated: no apparent potential for increased risk was reported and most of CCV adverse events with indacaterol occurred in patients with pre-existing cardiovascular risk factors [76]. Similarly, cardiovascular safety was specifically assessed for LAMAs, which are known to be associated with cardiovascular adverse events, such as arrhythmias [77]. Tiotropium did not increase the risk of cardiac events, mortality and serious adverse events even in patients who experienced recent myocardial infarction, heart failure, and unstable rhythm disorders [78]. Glycopyrronium and tiotropium showed similar long-term safety in clinical trials and post-marketing surveillance period, without increasing CCV compared to placebo [79], as well as all approved LAMA/LABA fixed-dose combinations had similar cardiovascular safety as single agents. It should be considered that the high prevalence of comorbidities in COPD patients makes poorly reproducible the pictures of clinical trials, usually performed on highly selected populations [80]. A recent meta-analysis on 23,168 COPD patients confirmed that all LAMA/LABA combinations were always more effective than LAMA or LABA alone in improving trough FEV1, without increasing the cardiovascular risk [81].

COPD per se increases the risk of cardiovascular diseases, such as angina, arrhythmias, congestive heart failure, and myocardium infarction [66], and heart failure is a strong independent predictor of all-cause mortality in COPD patients [82]. The presence of COPD affects the choice of pharmacological treatment for concomitant heart failure: per instance, $\beta$-blockers are less frequently used in COPD patients, than in general population [83]. However, based on retrospective studies, the pooled relative risk of COPD-related mortality secondary to $\beta$-blocker use was 0.69 (95\% CI: $0.62-0.78 ; \mathrm{I} 2=82 \%)$ [84] and some authors reported that $\beta$-blockers may reduce exacerbations in patients with both mild and 
severe disease $[85,86]$. $\beta$-blockers more selective for the $\beta(1)$-AR are more appropriate for COPD patients, with an attentive monitoring of lung function and symptoms [87]. Prospective real-world clinical studies on $\beta$-blockers in COPD patients should be recommended.

In addition to traditional therapies targeted on lungs, newer strategies potentially able to affect and mitigate cardiovascular risks in patients with COPD should be considered: angiotensin-converting enzyme inhibitors and angiotensin II type 1 receptor blockers, and $\beta$ adrenoceptor blockers, in addition to their benefit on treating comorbidities, might affect COPD [75], and early data suggest that may improve survival and reduce hospitalization, due to acute exacerbations of COPD [51].

\subsection{COPD, depression, and cognitive dysfunction}

Over $40 \%$ of elderly patients with COPD show clinically significant depressive symptoms that may warrant medical intervention and interfere in their daily activities and self-management of respiratory condition [88]. Long-term studies suggest that there is a bidirectional relationship between COPD and depression: COPD persistently increases the risk of depression and, the presence of depression contributes to worsen COPD course and affect the response during exacerbations [89]. From a molecular point of view, developing depressive symptoms and excessive fatigue that interfere in daily activities were associated with systemic inflammation and increased levels of tumor necrosis factor alpha (TNF $\alpha$ ) [89]. However, the exact mechanism or pathway by which COPD patients develop depression is still uncertain. It is most likely the complex interaction of physical, behavioral, physiological and systemic inflammation that may contribute to an increased risk of depressive symptoms. Short-term intervention studies suggest that both pulmonary rehabilitation and cognitive behavioral therapies are beneficial in improving depressive symptoms and quality of life, but 
the long-term efficacy of these approaches is unknown [90-92].

Cognitive functions should be evaluated before developing a self-management plan to comply with patient's needs and skills, since these factors can directly affect the adherence to treatment [93]. Tulek et al. [93] showed a negative association between cognitive function and number of exacerbations: COPD exacerbations increased $\mathrm{C}$ reactive protein (CRP) levels, fibrinogen, and leukocytes and this systemic inflammation impaired the cognitive abilities required to perform the MMSE (Mini Mental State Examination) and the BrownPeterson tests [93]. The MMSE test is commonly used in almost all clinical domains and it may be useful also for COPD patients [93]. Once evaluated the cognitive function, medications and therapies should be systematically reviewed to optimize the adherence and avoid adverse effects.

The high incidence of comorbidities in COPD patients implies the combination of multiple drugs, with an increased risk of adverse events. Molecules and schedules should be attentively chosen and monitored to immediately identify a drug-related symptom [94]. The risk associated to polytherapy is particularly high for elderly subjects, as COPD patients commonly are. Therefore, it is important to determine how healthy the elderly subject is through validated and suitable parameters, and to identify those patients who could benefit from a combined approach for COPD and comorbidities.

\subsection{EIderly, COPD and frailty}

Elderly patients with COPD are often defined as frail subjects $[95,96]$. Frailty is a progressive physiological decline in multiple organ systems marked by loss of function, loss of physiological reserve and increased vulnerability to disease [97]. Biological (inflammation and loss of hormones), clinical (e.g. sarcopenia and osteoporosis) and social factors are involved in frailty onset, evolution and prognosis $[98,99]$ and should be considered in COPD 
patient management. Frailty and respiratory impairment are strongly related and substantially increase the risk of death, when both are present [100].

Gait speed is a key marker of frailty: in the COPD context, it may capture many systemic effects of disease severity, rather than pulmonary function assessment alone, and it may reflect the global-wellbeing of subject. The gait speed is commonly assessed by exercise capacity in the 6-minute walk distance test in both clinical trial, as an outcome of lung function, and daily practice, as an indicator of lung functional capacity [101]. The gait speed decreases with COPD severity and is associated with age, clinical symptoms, pulmonary functions, and quality of life scores [100]. Standardizing the gait speed test may be important to obtain normative gait speed data, define their relationship with morbidity and mortality rates, and compare the effects of different treatment modalities, such as supplemental oxygen and corticosteroids [102].

Another useful test to assess the performance status in COPD patients is the Short Physical Performance Battery (SPPB). The SPPB includes results from three component tests of functional relevance: standing balance, 4-meter gait speed (4MGS), and five-repetition sitto-stand motion (5STS). Each component is scored based on a subscale and the three subscores are summed to obtain the final score. The SPPB has been validated for assessing mobility limitations in COPD patients and its results have significantly associated with the ability to perform physical activities in daily living, such as changing and maintaining body position, carrying, moving and handling objects, or walking [103]. Patients with COPD should be screened with SPPB to early identify subjects with limited mobility who may require supplemental rehabilitation strategies to prevent disability and restore normal function [103].

Therefore, SPPB, 6-minute walk distance test and lung function assessment, all 
together, are useful parameters to build a complete picture of a patient and propose an appropriate therapeutic intervention, because identifying the link between frailty, dyspnea, and chronic respiratory diseases represents a novel and practical approach to manage COPD patients. An increase in functional capacity, measured by gait speed or SPPB, may be an interesting target for therapy in frail elderly COPD subjects.

\subsection{COPD and osteoporosis}

The prevalence of osteoporosis in COPD patients varies between 9-69\%, depending on diagnostic methods, population, and severity of underlying respiratory disease, and is assumed to be two- to fivefold higher than counterparts without airflow obstruction [104]. COPD and osteoporosis share common risk factors, including past or current tobacco smoking, inactivity, and cachexia that are mainly associated with a decreased bone mineral density [105]; COPD itself is an independent predictor of bone mineral density reduction and increased risk of vertebral fractures [106]. COPD patients should be encouraged to avoid bone loss, recommending physical exercises, when feasible, rehabilitation programs, and counselling on fall prevention [107]. In the COPD management, corticosteroids should be limited and replaced with other therapies which favor bronchodilation and prevent exacerbation. During oral glucocorticosteroids treatment, a concomitant antiresorptive therapy should be considered, because bone loss starts immediately, in a daily dose relationship [107].

\subsection{COPD and A1AT deficiency}

Systemic deficiency of alpha-1 antitrypsin (A1AT) leads to an increase susceptibility to chronic inflammatory conditions, including COPD. A1AT deficiency underlies approximately $5 \%$ of all cases of COPD [108]. In these patients, the augmentation therapy with plasma-derived A1AT reduces the rate of lung function decline and the frequency of 
exacerbations, and shows significant benefit in subjects having FEV1 in the range of 35-49\% of predicted normal [109].

\subsection{Cachexia and malnutrition in COPD}

Cachexia is a complex syndrome frequently present in COPD patients and is associated with increased mortality, poor health-related quality of life, and muscle weakness [110]. The clinical phenotype of cachexia ranges from minimal or no weight loss, with signs of muscle wasting (e.g. anorexia, inflammation) to severe weight loss, muscle depletion, fatigue, and reduced mobility [111]. In patients with COPD and either overt cachexia or minimal weight loss, early nutritional support has shown clinical benefits on body weight, fat-free mass index, exercise tolerance, and respiratory and non-respiratory muscle strength, without safety issues [111].

\subsection{Conclusion}

A number of COPD patients have concomitant morbidities. Some of them may be independent of COPD and result from aging process, some may be direct consequences of COPD, and some may be caused by the same risk factors, mainly smoking. Irrespective of the links between COPD and comorbidities, physicians must be aware of their existence to make a suitable therapeutic plan for each individual patient. Pulmonary function testing remains the cornerstone for diagnosis, but it should not be always limited to simple spirometry. Bronchodilation is the first-line treatment for the majority of COPD patients even in the presence of comorbidities, which must be treated appropriately, keeping in mind possible drug interactions.

\subsection{Expert commentary}

The presence of multiple comorbidities in COPD patients complicates the clinical management of the disease. The goals in this field are to personalize all the therapeutic plan, 
from diagnosis to treatment, and to consider the peculiar characteristics of each patient. Firstly, an accurate assessment of respiratory function should be performed, and combining clinical and functional data will allow distinguishing patients with prevalent emphysema from those with prevalent chronic bronchitis. In addition to lung function assessment, other tests, including the Short Physical Performance Battery, the 6-minute walk distance test, the Mini Mental State Examination may be useful to obtain a complete picture of patient functioning and to propose the most appropriate therapeutic intervention. Similarly, depressive symptoms or malnutrition and cachexia should be recognized at early stage and promptly managed. Therefore, identifying the link between frailty, comorbidities, and chronic respiratory diseases will represent the most effective practical approach to diagnose and manage COPD patients, especially elderly ones. In presence of comorbidities, their treatment may prolong survival and impact on health care costs, unlike COPD treatments that may improve symptoms and decrease exacerbations, but does not prevent progression nor increase survival. Thus, the therapeutic plan of COPD should consider the patient as a whole and all interventions should be aimed to improve the overall health status and to treat or, at least, do not worsen comorbidities. Careful attention should be focused on patients with coexisting cardiovascular conditions and cardiovascular safety should be monitored during COPD therapy since bronchodilators -both LAMAs and LABAs- have a high potential to impact on cardiovascular activity, COPD per se increases the cardiovascular risk, and longterm treatment for previous cardiovascular diseases may be ongoing. Furthermore, the high incidence of comorbidities in COPD patients implies the combination of multiple drugs, with an increased risk of adverse events. Molecules and schedules should be attentively chosen and monitored to avoid drug- drug interactions and to immediately identify drug-related symptoms. 
Therefore, a future challenge will be to identify for each patient a personalized and multidimensional approach that will consider the patient as a whole. In the management of patients with COPD the goal that need to be achieved is the strong collaboration of key areas that include pulmonary medicine, geriatrics and cardiology.

Complete pulmonary profile, based on age, gender and the presence of comorbidity along with additional functional parameters to be validated are necessary for a proper assessment of the complexity of this kind of patient.

At present, the areas of interest of research are focused on COPD diagnosis and pharmacodynamic interactions between treatments, to both manage COPD and address specific comorbidities, and further deepen the role of inflammation in COPD pathogenesis. Establishing the correct relationship between COPD and inflammation and aging would contribute to further improve clinical outcomes of patients and develop strategies to prevent the disease. Cardiovascular safety will be another point of clinical importance in COPD patient management and research in this field.

In the coming years, the majority of patients with COPD will be elderly, hill, and frail. It will be important to adapt current strategies to this population or identify novel approaches through validated and suitable parameters.

\subsection{Five-year view}

In 5 years, the COPD landscape will change; further improvement will be done to assess a complete pulmonary profile and to address comorbidities. Therapies will be personalized, taking advantage of a wide availability of drugs, with well differentiated pharmacological characteristics. 


\section{Key issues}

- COPD is characterized by a slowly progressive deterioration in lung function, mostly becoming evident with aging. COPD is frequently associated with other age-related morbid conditions, which may exist independently, or as consequences of COPD, or because they share common risk factors.

- Pulmonary function testing remains the cornerstone for diagnosis, but it should not be always limited to simple spirometry. Indeed, spirometric measurements are good survival predictors, but loosely correlated with patient-centered outcomes, such as dyspnea, quality of life, exercise tolerance.

- In the overall management of COPD patients, the assessment of comorbidities is a valuable tool to improve prognosis and quality of life: the patient is currently considered as a whole, in whom COPD and other comorbidities synergize to define the health status.

- Both acute and chronic lung inflammation contributes to cardiovascular morbidity in COPD patients.

- Cardiovascular safety should be monitored during COPD therapy for many reasons: both LAMAs and LABAs have a high potential to impact on cardiovascular activity; COPD per se increases the cardiovascular risk; some patients may be on long-term treatment for previous cardiovascular diseases.

- In the treatment of patients with COPD, clinicians must look beyond the lung and develop therapeutic approaches to prevent or mitigate cardiovascular risks associated with COPD. 


\section{Funding}

This manuscript has received funding from Novartis Farma SpA (Origgio - IT).

\section{Declaration of interests}

A Corsini has received sponsorship by AstraZeneca, Sankyo, Pfizer, Bristol-Meyers Squibb and Mylan. The authors have no other relevant affiliations or financial involvement with any organization or entity with a financial interest in or financial conflict with the subject matter or materials discussed in the manuscript apart from those disclosed. Peer reviewers on this manuscript have no relevant financial or other relationships to disclose.

\section{Acknowledgements}

Editorial support was provided by Content Ed Net, with the helpful contribution in drafting the text by Elisa Sala, PhD Medical Writer. 


\section{References}

Reference annotations

* Of interest

** Of considerable interest

1. Burrows B, Fletcher CM, Heard BE, et al. The emphysematous and bronchial types of chronic airways obstruction. A clinicopathological study of patients in London and Chicago. Lancet. 1966;1:830-835.

2. Snider GL. Chronic obstructive pulmonary disease: a definition and implications of structural determinants of airflow obstruction for epidemiology. Am Rev Respir Dis. $1989 ; 140: \mathrm{S3}-8$.

3. Pauwels RA, Buist AS, Calverley PM, et al. Global strategy for the diagnosis, management, and prevention of chronic obstructive pulmonary disease. NHLBI/WHO Global Initiative for Chronic Obstructive Lung Disease (GOLD) Workshop summary. Am J Respir Crit Care Med. 2001;163:1256-1276.

4. Rabe KF, Hurd S, Anzueto A, et al. Global strategy for the diagnosis, management, and prevention of chronic obstructive pulmonary disease: GOLD executive summary. Am J Respir Crit Care Med. 2007;176:532-555.

5. Vogelmeier CF, Criner GJ, Martinez FJ, et al. Global Strategy for the Diagnosis, Management, and Prevention of Chronic Obstructive Lung Disease 2017 Report. GOLD Executive Summary. Am J Respir Crit Care Med. 2017;195:557-582.

6. Bush A. Lung Development and Aging. Ann Am Thorac Soc. 2016:S438-S446. doi: 10.1513/AnnalsATS.201602-112AW.

7. Hunninghake GM, Cho MH, Tesfaigzi Y, et al. MMP12, lung function, and COPD in high-risk populations. N Engl J Med. 2009;361:2599-2608.

8. Pillai SG, Ge D, Zhu G, et al. A genome-wide association study in chronic obstructive pulmonary disease (COPD): identification of two major susceptibility loci. PLoS Genet. 2009;5:e1000421. 
9. Hogg JC. Pathophysiology of airflow limitation in chronic obstructive pulmonary disease. Lancet. 2004;364:709-721.

10. Yawn BP, Kaplan A. Co-morbidities in people with COPD: a result of multiple diseases, or multiple manifestations of smoking and reactive inflammation? Prim Care Respir J. 2008;17:199-205.

*This is an interesting paper about the relationship between COPD and comorbidities.

11. Fabbri LM, Luppi F, Beghé B, et al. Complex chronic comorbidities of COPD. Eur Resp J. 2008;31:204-212.

12. Agusti A. Thomas A. Neff lecture. Chronic obstructive pulmonary disease: a systemic disease. Proc Am Thorac Soc. 2006;3:478-481.

**This paper provides an overview about COPD as a systemic disease.

13. Barr RG, Bluemke DA, Ahmed FS, et al.Percent emphysema, airflow obstruction, and impaired left ventricular filling. N Engl J Med. 2010;362:217-227.

14. O'Reilly J, Jones MM, Parnham J, et al. Management of stable chronic obstructive pulmonary disease in primary and secondary care: summary of updated NICE guidance. BMJ. 2010;340:c3134. doi: 10.1136/bmj.c3134.

15. O'Donnell DE, Hernandez P, Kaplan A, et al. Canadian Thoracic Society recommendations for management of chronic obstructive pulmonary disease 2008 update - highlights for primary care. Can Respir J. 2008;15 Suppl A:1A-8A.

16. McKenzie DK, Frith PA, Burdon JG, et al. The COPDX Plan: Australian and New Zealand Guidelines for the management of Chronic Obstructive Pulmonary Disease 2003. Med J Aust. 2003;178 Suppl:S7-39.

17. Niewoehner DE, Kleinerman J, Rice DB. Pathologic changes in the peripheral airways of young cigarette smokers. N Engl J Med. 1974;291:755-758.

18. Baldi S, Miniati M, Bellina CR, et al. Relationship between extent of pulmonary emphysema by high-resolution computed tomography and lung elastic recoil in 
patients with chronic obstructive pulmonary disease. Am J Respir Crit Care Med. 2001;164:585-589.

19. Quanjer PH, Cooper B, Ruppel GL, et al. Defining airflow obstruction. Eur Respir J. 2015;45:561-562.

20. Quanjer PH, Enright PL, Miller MR, et al. The need to change the method for defining mild airway obstruction. Eur Respir J. 2011;37:720-2.

21. Quanjer PH, Pretto JJ, Brazzale DJ, et al. Grading the severity of airways obstruction: new wine in new bottles. Eur Respir J. 2014;43:505-512.

22. Pellegrino R, Crimi E, Gobbi A, et al. Severity grading of chronic obstructive pulmonary disease: the confounding effect of phenotype and thoracic gas compression. J Appl Physiol. 2015;118:796-802.

23. Çolak Y, Marott JL, Vestbo J, et al. Overweight and obesity may lead to underdiagnosis of airflow limitation: findings from the Copenhagen City Heart Study. COPD. 2015;12:5-13.

24. Enright PL, Brusasco V. Obesity and risks of misclassification and over-treatment in COPD. COPD. 2015;12:3-4.

25. Landbo C, Prescott E, Lange $P$, et al. Prognostic value of nutritional status in chronic obstructive pulmonary disease. Am J Respir Crit Care Med. 1999;160:1856-1861.

26. Bates DV, Woolf CR, Paul GI. A report on the first two stages of the co-ordinated study of chronic bronchitis in the Department of Veterans Affairs, Canada. Med Serv J Can. 1962;18:211-303.

27. Bates DV, Gordon CA, Paul GI, et al. Chronic bronchitis. Report on the third and fourth stages of the co-ordinated study of chronic bronchitis in the Department of Veterans Affairs, Canada. Med Serv J Can. 1966;22:1-59.

28. Mannino DM, Doherty DE, Sonia Buist A. Global Initiative on Obstructive Lung Disease (GOLD) classification of lung disease and mortality: findings from the 
Atherosclerosis Risk in Communities (ARIC) study. Respir Med. 2006;100:115122.

29. Pellegrino R, Viegi G, Brusasco V, et al. Interpretative strategies for lung function tests. Eur Respir J. 2005;26:948-968.

30. Mannino DM, Diaz-Guzman E, Buist S. Pre- and post-bronchodilator lung function as predictors of mortality in the Lung Health Study. Respir Res. 2011;12:136.

31. Curtis JR, Deyo RA, Hudson LD. Pulmonary rehabilitation in chronic respiratory insufficiency. 7. Health-related quality of life among patients with chronic obstructive pulmonary disease. Thorax. 1994;49:162-170.

32. O'Donnell DE. Hyperinflation, dyspnea, and exercise intolerance in chronic obstructive pulmonary disease. Proc Am Thorac Soc. 2006;3:180-184.

33. Athanazio R. Airway disease: similarities and differences between asthma, COPD and bronchiectasis. Clinics 2012;67:1335-1343.

34. Camiciottoli G, Bigazzi F, Paoletti M, et al. Pulmonary function and sputum characteristics predict computed tomography phenotype and severity of COPD. Eur Respir J. 2013;42:626-35.

35. Agusti A, Calverley PM, Celli B, et al. Characterisation of COPD heterogeneity in the ECLIPSE cohort. Respir Res. 2010;11:122.

36. Berry CE, Wise RA. Mortality in COPD: causes, risk factors, and prevention. COPD 2010;7:375-382.

37. McGarvey LP, John M, Anderson JA, et al. Ascertainment of cause-specific mortality in COPD: operations of the torch clinical endpoint committee. Thorax 2007;62:411-415.

38. Divo M, Cote $\mathrm{C}$, de Torres $\mathrm{J}$, et al. Comorbidities and risk of mortality in patients with chronic obstructive pulmonary disease. Am J Respir Crit Care Med. 2012;186:155-161. 
*This paper describes an algorithm to evaluate the role of comorbidities in COPD patients

39. Vanfleteren L, Spruit M, Groenen M, et al. Clusters of comorbidities based on validated objective measurements and systemic inflammation in patients with chronic obstructive pulmonary disease. Am J Respir Crit Care Med. $2013 ; 187: 728-735$.

*This paper describes an algorithm to evaluate the role of comorbidities in COPD patients

40. Luppi F, Franco F, Beghé B, et al. Treatment of chronic obstructive pulmonary disease and its comorbidities. Proc Am Thorac Soc. 2008;5:848-856.

41. Durham AL, Caramori G, Chung KF, et al. Targeted anti-inflammatory therapeutics in asthma and chronic obstructive lung disease. Transl Res. 2016;167:192-203.

* This paper describes novel therapeutic approaches with anti-inflammatory drugs in COPD

42. Battram C, Charlton SJ, Cuenoud B, et al. In vitro and in vivo pharmacological characterization of 5-[(R)-2-(5,6-diethyl-indan-2-ylamino)-1-hydroxy-ethyl]-8hydroxy-1H-quinolin-2-one (indacaterol), a novel inhaled $\beta(2)$ adrenoceptor agonist with a 24-h duration of action. J Pharmacol Exp Ther. 2006;317:762-770.

43. Tashkin DP, Fabbri LM. Long-acting $\beta$-agonists in the management of chronic obstructive pulmonary disease: current and future agents. Respir Res. 2010;11:149.

44. Belmonte KE. Cholinergic pathways in the lungs and anticholinergic therapy for chronic obstructive pulmonary disease. Proc Am Thorac Soc. 2005;2:297-304.

45. Matera MG, Cazzola M. Muscarinic Receptor Antagonists. Handb Exp Pharmacol. 2016 Oct 28. 
46. Wedzicha JA, Banerji D, Chapman KR, et al. Indacaterol-Glycopyrronium versus Salmeterol-Fluticasone for COPD. N Engl J Med. 2016;374:2222-2234.

47. Roche N, Chapman KR, Vogelmeier CF, et al. Chronic Obstructive Pulmonary Disease Treatment. Data from the FLAME Trial. Am J Respir Crit Care Med. 2017;195:1189-1197.

48. Rogliani P, Calzetta L, Cazzola M, Matera MG. Drug safety evaluation of roflumilast for the treatment of COPD: a meta-analysis. Expert Opin Drug Saf. 2016 Aug;15(8):1133-46.

49. Cazzola M, Calzetta L, Rogliani P, Matera MG. The discovery of roflumilast for the treatment of chronic obstructive pulmonary disease. Expert Opin Drug Discov. 2016 Jul;11(7):733-44).

50. Albert R, Connett J, Bailey W, et al. Azithromycin for Prevention of Exacerbations of COPD. N Eng1 J Med. 2011;365:689-698.

51. Cazzola M, Calzetta L, Rinaldi B, et al. Management of Chronic Obstructive Pulmonary Disease in Patients with Cardiovascular Diseases. Drugs. 2017 doi: $10.1007 / \mathrm{s} 40265-017-0731-3$.

** This paper highlights the potential impact of traditional therapies for COPD that are centred on treating the lungs and newer strategies potentially able to affect and mitigate cardiovascular risks in patients with COPD.

52. Campo G, Pavasini R, Biscaglia S, et al. Overview of the pharmacological challenges facing physicians in the management of patients with concomitant cardiovascular disease and chronic obstructive pulmonary disease. Eur Heart J Cardiovasc Pharmacother. 2015;1:205-211.

* This paper discusses the challenges in managing COPD patients with cardiovascular condition. 
53. Miravitlles M, Soler-Cataluña JJ, Calle M, et al. A new approach to grading and treating COPD based on clinical phenotypes: summary of the Spanish COPD guidelines (GesEPOC). Prim Care Respir J. 2013;22:117-121.

54. Sundh J, Johansson G, Larsson K, et al. The phenotype of concurrent chronic bronchitis and frequent exacerbations in patients with severe COPD attending Swedish secondary care units. Int J Chron Obstruct Pulmon Dis. 2015;10:23272334.

55. Cazzola M, Ciaprini C, Page CP, et al. Targeting systemic inflammation: novel therapies for the treatment of chronic obstructive pulmonary disease. Expert Opin Ther Targets. 2007;11:1273-1286.

56. Sanguinetti CM. N-acetylcysteine in COPD: why, how, and when? Multidiscip Respir Med. 2016;11:8.

57. Matera MG, Calzetta L, Cazzola M. Oxidation pathway and exacerbations in COPD: the role of NAC. Expert Rev Respir Med. 2016;10:89-97.

58. Cazzola M, Calzetta L, Page C, Jardim J, Chuchalin AG, Rogliani P, Matera MG. Influence of $\mathrm{N}$-acetylcysteine on chronic bronchitis or COPD exacerbations: a meta-analysis. Eur Respir Rev. 2015 Sep;24(137):451-61.

59. Criner GJ, Bourbeau J, Diekemper RL, et al. Prevention of acute exacerbations of COPD. American College of Chest Physicians and Canadian Thoracic Society Guideline. Chest. 2015;147:894-942.

60. Beghè $\mathrm{S}$, Verdure $\mathrm{A}$, Roca $\mathrm{M}$, et al. Exacerbation of respiratory symptoms in COPD patients may not be exacerbations of COPD. Eur Resp J. 2013;41:993-995.

61. Jessup M, Abraham WT, Casey DE, et al. 2009 focused update: ACCF/AHA Guidelines for the Diagnosis and Management of Heart Failure in Adults: a report of the American College of Cardiology Foundation/ American Heart Association Task Force on Practice Guidelines: developed in collaboration with the International Society for Heart and Lung Transplantation. Circulation. 2009;119: $1977-2016$. 
62. Task Force for Diagnosis and Treatment of Acute and Chronic Heart Failure 2008 of European Society of Cardiology. ESC Guidelines for the diagnosis and treatment of acute and chronic heart failure 2008: the Task Force for the Diagnosis and Treatment of Acute and Chronic Heart Failure 2008 of the European Society of Cardiology. Developed in collaboration with the Heart Failure Association of the ESC (HFA) and endorsed by the European Society of Intensive Care Medicine (ESICM). Eur Heart J. 2008;29:2388-2442.

63. Freeborne N, Lynn J, Desbiens NA. Insights about dying from the SUPPORT project. The study to understand prognoses and preferences for outcomes and risks of treatments. J Am Geriatr Soc. 2000;48: S199-205.

64. Holguin F, Folch E, Redd SC, et al. Comorbidity and mortality in COPD-related hospitalizations in the United States, 1979 to 2001. Chest. 2005;128:2005-2011.

65. Huiart L, Ernst P, Suissa S. Cardiovascular morbidity and mortality in COPD. Chest. 2005; 128:264-266.

66. Curkendall SM, Lanes S, de Luise C, et al. Chronic obstructive pulmonary disease severity and cardiovascular outcomes. Eur J Epidemiol. 2006; 21:803.813.

67. Fabbri LM, Rabe KF. From COPD to chronic systemic inflammatory syndrome? Lancet 2007;370:797-799.

68. Clayton TC, Thompson M, Meade TW. Recent respiratory infection and risk of cardiovascular disease: case-control study through a general practice database. Eur Heart J. 2008;29:96-103.

69. Fabbri LM, Calverley PM, Izquierdo-Alonso JL, et al. M2-127 and M2-128 study groups. Roflumilast in moderate-to-severe chronic obstructive pulmonary disease treated with long-acting bronchodilators: two randomised clinical trials. Lancet 2009;374:695-703.

70. Calverley PM, Rabe KF, Goehring UM, et al. M2-124 and M2-125 study groups. Roflumilast in symptomatic chronic obstructive pulmonary disease: two randomised clinical trials. Lancet 2009;374:685-694. 
71. Cazzola M, Rogliani P, Matera MG. Cardiovascular disease in patients with COPD. Lancet Respir Med. 2015;3:593-595.

72. Campo G, Pavasini R, Malagù M, et al. Chronic obstructive pulmonary disease and ischemic heart disease comorbidity: overview of mechanisms and clinical management. Cardiovasc Drugs Ther. 2015;29:147-157.

73. Roversi S, Roversi P, Spadafora G, et al. Coronary artery disease concomitant with chronic obstructive pulmonary disease. Eur J Clin Invest. 2014;44:93-102.

74. Van Eeden S, Leipsic J, Paul Man SF, et al. The relationship between lung inflammation and cardiovascular disease. Am J Respir Crit Care Med. 2012;186:11-16.

75. Cazzola M, Calzetta L, Matera MG, et al. Chronic obstructive pulmonary disease and coronary disease: COPDCoRi, a simple and effective algorithm for predicting the risk of coronary artery disease in COPD patients. Respir Med. 2015;109:10191025 .

76. Worth H, Chung KF, Felser JM, et al. Cardio- and cerebrovascular safety of indacaterol vs formoterol, salmeterol, tiotropium and placebo in COPD. Respir Med. 2011;105:571-579.

77. Sharafkhaneh A, Majid H, Gross NJ. Safety and tolerability of inhalational anticholinergics in COPD. Drug Healthc Patient Saf. 2013;5:49-55.

78. Tashkin DP, Leimer I, Metzdorf N, et al. Cardiac safety of tiotropium in patients with cardiac events: a retrospective analysis of the UPLIFT ${ }^{\circledR}$ trial. Respir Res. $2015 ; 16: 65$.

79. D'Urzo AD, Kerwin EM, Chapman KR, et al. Safety of inhaled glycopyrronium in patients with COPD: a comprehensive analysis of clinical studies and postmarketing data. Int J Chron Obstruct Pulmon Dis. 2015;10:1599-1612.

80. Matera MG, Rogliani P, Calzetta L, et al. Safety Considerations with Dual Bronchodilator Therapy in COPD: An Update. Drug Saf. 2016;39:501-508. 
81. Calzetta L, Rogliani P, Matera MG, et al. A systematic review with meta-analysis of dual bronchodilation with LAMA/LABA for the treatment of stable COPD. Chest. 2016;149:1181-1196.

82. Boudestein LC, Rutten FH, Cramer MJ, et al. The impact of concurrent heart failure on prognosis in patients with chronic obstructive pulmonary disease. Eur J Heart Fail. 2009;11:1182-1188.

83. Hawkins NM, Jhund PS, Simpson CR, et al. Primary care burden and treatment of patients with heart failure and chronic obstructive pulmonary disease in Scotland. Eur J Heart Fail. 2010;12:17-24.

84. Etminan M, Jafari S, Carleton B, et al. B-blocker use and COPD mortality: a systematic review and meta-analysis. BMC Pulm Med. 2012;4:12-48.

85. Bhatt S, Wells J, Kinney G, et al. B-blockers are associated with a reduction in COPD exacerbations. Thorax 2016;71:8-14.

86. Farland MZ, Peters CJ, Williams JD, et al. $\beta$-Blocker use and incidence of chronic obstructive pulmonary disease exacerbations. Ann Pharmacother. 2013;47:651656.

87. Matera MG, Martuscelli E, Cazzola M. Pharmacological modulation of $\beta$ adrenoceptor function in patients with coexisting chronic obstructive pulmonary disease and chronic heart failure. Pulm Pharmacol Ther. 2010;23:1-8.

88. Yohannes A., Baldwin R., Connolly M. Mood disorders in elderly patients with chronic obstructive pulmonary disease. Rev Clin Geront 2000;10:193-202.

89. Connolly MJ, Yohannes AM. The impact of depression in older patients with chronic obstructive pulmonary disease and asthma. Maturitas. 2016;92:9-14.

90. Coventry PA, Hind D. Comprehensive pulmonary rehabilitation for anxiety and depression in adults with chronic obstructive pulmonary disease: systematic review and meta-analysis. J Psychsom Res. 2007;63:551-565. 
91. Smith SM, Sonego S, Ketcheson L, et al. A review of the effectiveness of psychological interventions used for anxiety and depression in chronic obstructive pulmonary disease. BMJ Open Respir Res. 2014;1:e00042.

92. Farver-Vestergaard I, Jacobsen D, Zachariae R. Efficacy of psychosocial interventions on psychological and physical health outcomes in chronic obstructive pulmonary disease: a systematic review and meta-analysis. Psychother Psychosom. 2015;84:37-50.

93. Tulek B, Atalay NB, Yildirim G, et al. Cognitive function in chronic obstructive pulmonary disease: Relationship to global initiative for chronic obstructive lung disease 2011 categories. Respirology. 2014;19:873-880.

94. Field T, Gurwitz J, Avorn J, et al. Risk factors for adverse drug events among nursing home residents. Arch Intern Med. 2001;161:1629-1634.

95. Faner R, Cruz T, Lopez-Giraldo A, et al. Network medicine, multimorbidity and the lung in the elderly. Eur Respir J. 2014;44:775-788.

96. Bousquet J, Dinh-Xuan A, Similowski T, et al. Should we use gait speed in COPD, FEV1 in frailty and dyspnoea in both? Eur Respir J. 2016;48:315-319.

97. Rodriguez-Manas L, Fried LP. Frailty in the clinical scenario. Lancet. 2015;385: e7-e9.

98. Morley JE, Vellas B, van Kan GA, et al. Frailty consensus: a call to action. J Am Med Dir Assoc. 2013;14:392-397.

99. Gobbens RJ, van Assen MA, Luijkx KG, et al. Determinants of frailty. J Am Med Dir Assoc. 2010;11:356-364.

100. Vaz Fragoso CA, Enright PL, McAvay G, et al. Frailty and respiratory impairment in older persons. Am J Med 2012;125:79-86.

101. Sciurba F, Criner G, Lee SM, et al. Six-minute walk distance in chronic obstructive pulmonary disease. Am J Respir Crit Care Med. 2003; 167:15221527. 
102. Ilgin D, Ozalevli S, Kilinc O, et al. Gait speed as a functional capacity indicator in patients with chronic obstructive pulmonary disease. Ann Thorac Med 2011;6:141-146.

103. Bernabeu-Mora R, Medina-Mirapeix F, Llamazares-Herrán E, et al. The Short Physical Performance Battery is a discriminative tool for identifying patients with COPD at risk of disability. Int J COPD. 2015;10:2619-2626.

104. Graat-Verboom L, Wouters EF, Smeenk FW, et al. Current status of research on osteoporosis in COPD: a systematic review. Eur Respir J. 2009;34:209-218.

105. Langhammer A, Forsmo S, Syversen U. Long-term therapy in COPD: any evidence of adverse effect on bone? Int J Chron Obstruct Pulmon Dis. 2009;4:365-380.

106. Lehouck A, Boonen S, Decramer M, et al. COPD, bone metabolism, and osteoporosis. Chest. 2011;139:648-657.

107. Mazokopakis EE, Starakis IK. Recommendations for Diagnosis and Management of Osteoporosis in COPD Men. ISRN Rheumatol. 2011;2011:901416.

108. Tuder RM, Janciauskiene SM, Petrache I. Lung disease associated with alpha1antitrypsin deficiency. Proc Am Thorac Soc. 2010;7:381-386.

109. Kueppers F. The role of augmentation therapy in alpha-1 antitrypsin deficiency. Curr Med Res Opin. 2011;27:579-588.

110. Muscaritoli M, Molfino A, Lucia S, et al. Cachexia: a preventable comorbidity of cancer. A T.A.R.G.E.T. approach. Crit Rev Oncol Hematol. 2015;94:251-259.

111. Calder PC, Laviano A, Lonnqvist F, et al. Targeted medical nutrition for cachexia in chronic obstructive pulmonary disease: a randomized, controlled trial. $\mathrm{J}$ Cachexia Sarcopenia Muscle. 2017 Sep 10. doi: 10.1002/jcsm.12228. [Epub ahead of print] 